\title{
La transformación digital, un desafío inmediato ocasionado por la pandemia de Covid-19 para las entidades del sector de educación superior
}

\author{
Digital transformation, an inmediate \\ challenge caused by the Covid-19 \\ pandemic for entities in the higher \\ education sector
}

\author{
Alfonso Rodríguez Ramirez ${ }^{1}$ \\ Jose Luis Garcia Molano² \\ Mateo Castrillón Peralta ${ }^{3}$ \\ Universidad Surcolombiana, Colombia
}

1 Docente Investigador Tiempo Completo Facultad de Economía y Administración de la Universidad Surcolombiana. Administrador de Empresas, Universidad Surcolombiana, Magíster en Ciencias de la Organización, Candidato a Doctor en Administración, Universidad del Valle. alfonso.rodriguez@usco.edu.co - Código https://orcid.org/0000-0001-9103-1969 Tel. +57 3222948593 - Colombia, Huila - Neiva

2 Investigador Universidad Surcolombiana. Programa Administración de Empresas de la Facultad de Economía y Administración de la Universidad Surcolombiana. u20161148483@usco.edu.co - Código https://orcid. org/0000-0002-9885-1860 - Tel. +57 3123397387 - Colombia, Huila - Neiva.

3 Investigador Universidad Surcolombiana. Programa Administración de Empresas de la Facultad de Economía y Administración de la Universidad Surcolombiana. u20161149661@usco.edu.co Código https://orcid. org/0000-0002-7481-2808 - Tel +57 3218496237 - Colombia, Huila - Neiva.

\section{RESUMEN}

Latransformación digital en la educación superior, aunque se considera un proceso que debería haberse llevado a cabo hace mucho tiempo en la adaptación a las tendencias del mundo moderno y las necesidades de sistematización de información, ha demostrado tener un rezago importante en Colombia, corroborando de manera consistente en la imposibilidad de mantener los niveles de investigación y de producción de conocimiento que se logran a partir de la formación tradicional presencial. Desde la revisión documental que ocupa el 
presente artículo se ha podido evidenciar que el concepto de transformación digital para la educación superior aparece sustancialmente relacionado con otros conceptos de necesario conocimiento para la modificación completa del sistema educativo, como son innovación social, innovación educativa, e inclusive sostenibilidad economica y financiera. El recorrido realizado permite establecer que el trabajo mancomunado y el conocimiento de las nuevas tendencias se reconocen como factores fundamentales en la promoción de una educación superior soportada en procesos de transformación digital, y que se construye con el apoyo de la totalidad de actores inmersos en el proceso.

\section{PALABRAS CLAVE:}

Transformación digital, educación superior, Covid-19.

\section{ABSTRACT}

The digital transformation in higher education, although it is considered a process that should have been carried out a long time ago in adapting to the trends of the modern world and the needs of information systematization, has shown to have an important lag in Colombia, demonstrated by consistently in the impossibility of maintaining the levels of research and knowledge production that are achieved from traditional face-to-face training. From the documentary review that this article occupies, it has been possible to show that the concept of digital transformation for higher education appears substantially related to other concepts of necessary knowledge for the complete modification of the educational system, such as social innovation, educational innovation, and even economic and financial sustainability. The route carried out allows us to establish that joint work and knowledge of new trends are recognized as fundamental factors in the promotion of higher education supported by digital transformation processes and that it is built with the support of all actors immersed in the process.

KEYWORDS: Digital transformation, higher education, Covid-19

\section{INTRODUCCIÓN}

La transformación digital, implica modificaciones constantes que requieren una dinámica y ejecución de diferentes procesos para optimizar las actividades o servicios que integra procesos y productos dentro de una organización. En efecto, la transformación digital se establece como el desarrollo de acciones que integran nuevas tecnologías en todas las áreas de una empresa o institución para mejorar sus oportunidades de estrategia gracias a la implementación de la tecnología (McKinsey, 2020).

En virtud de lo anterior, las empresas constantemente establecen como objetivo una mejora en los procesos, un fortalecimiento de su competitividad y la capacidad de poder crear un valor agregado, las entidades educativas no son ajenas a la consolidación de estos objetivos, como resultado, estas acciones requieren de la incorporación de dinámicas que denotan un cambio radical, asumiendo, que la transformación digital se concibe como un proceso que debe ser contemplado a corto plazo acorde con las capacidades y necesidades de cada institución.

Sin embargo, a raíz de la pandemia ocasionado por la COVID-19 y el estado de emergencia mundial, la transformación digital no se constituyó un desafío a corto plazo para cada una de las organizaciones, sino que adquirió la connotación de inmediatez, en consecuencia, fue tanto el impacto que la pandemia ocasionó que el PNUD (2020) refirió que la transformación digital que se hubiera llevado entre tres a cinco años se realizó en unos cuantos meses en el año 2020. En ese particular, las organizaciones del sector educativo tuvieron que afrontar una 
transición inmediata, pues antes de la pandemia los enfoques virtuales estaban establecidos en las agendas como procesos de aplicación a largo plazo, pues requerían de inversión, toma de decisiones asertivas, y capacitación a toda la comunidad educativa que no estaba dispuesto en la agenda de acciones a realizar en un periodo de tiempo cercano.

Es así, que las entidades del sector de educación de Colombia, tuvieron que enfrentar adicional a los cambios suscitados por el confinamiento y el distanciamiento, la adopción de unos procesos digitales para sustentar cada una de sus actividades de modo virtual, pues las condiciones de aislamiento social no permitían que las entidades realicen sus actividades de forma como acostumbraban centrados en impartir los diferentes contenidos académicos en espacios físicos acordes a las necesidades del proceso de aprendizaje.

A partir de ello, el presente artículo presenta un análisis que permite identificar como la pandemia estipuló la transformación digital como un desafío inmediato, situación que puso en evidencia que las entidades del sector de educación en la ciudad que aún no habían iniciado el proceso transformador tuvieron que realizarlo cuanto antes, pues esta era una de las pocas estrategias que estas entidades tenían para enfrentar los retos que imponía la pandemia, sumado a la incertidumbre generalizada que originaba la misma.

Conviene mencionar, que desde la perspectiva pedagógica la transformación digital ha adquirido una papel protagonista, pues es indispensable identificar como se ha llevado a cabo la respuesta de las entidades dedicadas a este sector ante la presencia de estos eventos disruptivos, y como se han podido canalizar mediante la implementación de acciones dirigidas a la transformación digital, teniendo en cuenta que la pandemia por Covid-19 se constituyó un acelerador de la transformación digital en cada una de ellas.

El análisis, se realiza reconociendo previamente cómo las entidades del sector educativo se han constituido como una de las que se han visto más afectadas por los cambios producidos por la pandemia, pues éstas tuvieron que implementar de manera inmediata una evolución que permita que la oferta académica que se presentaba de manera presencial pueda seguir operando de modo virtual a pesar de las circunstancias, incluyendo aspectos que para muchas instituciones eran ajenas a estrategias como la b-learning, la digitalización de contenidos y servicios, y la adaptación de contenidos educativos a un entorno netamente virtual.

Estos cambios, establecieron a la tecnología como un gran aliado, empero, se identificaron una serie de inconvenientes no solo con la funcionalidad de las plataformas, sino también para los participantes activos de la misma que conforma el personal de las entidades del sector, como los docentes, estudiantes y personal administrativo, pues se propendió a que la comunidad educativa adquiera la transformación digital como un modelo que brinda un soporte a las actividades de aprendizaje, además de que se identifica como una medida que garantizara que los estudiantes tenga acceso al contenido académico que garantice su derecho universal a la educación.

En ese orden de ideas, el desafío inmediato de las entidades del sector de educación consistió en la toma de decisión de poder brindar clases de forma remota y continuar operando a pesar del confinamiento social, implicando unas modificaciones en diferentes aspectos, tanto desde la perspectiva de pedagogía, operación y funcionamiento, mantenimiento y procesos de digitalización que antes no estaban estipulados, así como la adopción de transformaciones digitales en elementos como la resolución de solicitudes y tramites, atención a usuarios 
y actividades de bienestar universitario, fundamental para salvaguardar los derechos de los estudiantes (Argudo, 2020)

En virtud de lo anterior, se identifica que dentro del contexto empresarial, las instituciones de educación superior tuvieron que enfrentar de manera intempestiva la transformación digital, es de esta manera que es relevante analizar como las entidades del sector en Colombia pusieron en marcha su transformación digital para permitir prestar el servicio de manera virtual, estableciendo un momento decisivo que implica retos y la incursión en uso y aplicación de herramientas digitales, inmersos en la ejecución de nuevos planes de acción que tienen en cuenta la flexibilización de contenidos académicos para las modalidades en la nube como el e-learning y b-learning.

En consecuencia, la pandemia por COVID-19 catapulto los temas de trasformación digital que las entidades del sector de educación en la ciudad no tenían tanta importancia, por consiguiente, el estado de emergencia facilito la iniciación de la transformación digital que ha sido adoptada no solo para solventar las necesidades de virtualidad, sino también para convertirse en un modelo emergente que brinda grandes bondades y beneficios para las organizaciones del sector educativo.

Finalmente, el objetivo del presente artículo radica en analizar como las entidades del sector de educación superior en Colombia asumieron la transformación digital como un desafío inmediato para poder continuar prestando el servicio de educación.

\section{METODOLOGÍA}

El presente artículo de investigación se realiza desde una búsqueda bibliográfica relacionada con los procesos digitales implementados en el entorno educativo, considerando tanto los elementos que se han impuesto como tendencia en el momento actual, así como los esfuerzos para generar una congruencia entre las necesidades de estudiantes y docentes y la implementación de los diferentes procesos pedagógicos que se han implementado en el entorno nacional. Con el fin anteriormente expuesto se ha estipulado la necesidad de ocupar diferentes buscadores documentales considerando como Redalyc, Scopus, Sciencedirect, Google Academic, repositorios institucionales, entre otros, teniendo en cuenta que los documentos a consultar deben ser bastante actuales y referirse de manera directa al entorno contextual propio de la pandemia por Covid-19, evento que tuvo lugar en el año 2020 a nivel mundial. En la misma medida la información recolectada se analizará a la luz de algunos elementos contextuales propios de la sociedad colombiana, que pueden ser determinantes para la construcción de un proceso digitalizado congruente con las necesidades de estudiantes y alumnos.

\section{RESULTADOS}

Para iniciar con la presente sección es necesario reconocer lo imperioso de recurrir a documentos oficiales de organismos internacionales que, en muchos casos, representan en buena medida no solo los condicionantes que son vividos por Colombia, sino también por América Latina y el mundo cuando a la situación de pandemia se refiere. Es preciso reconocer en ese sentido que, cuando se analiza la transformación digital en pro de la educación, específicamente en las Instituciones de Educación Superior ello implica necesariamente la realización de cambios profundos y coordinados no solo en los factores propios del entorno comunicacional, sino también en el entorno formativo; desde el punto de vista pedagógico, y de los actores de cambio como son el Estado, los docentes y los estudiantes y sus familias.

La pandemia por su condición de inmediatez ha permitido generar cambios suscitados en un periodo realmente corto, pero ha demostrado 
de manera consistente la necesidad de generar cambios estructurales. En efecto, bajo los elementos anteriormente mencionados, se construyen los principales factores que estructura la literatura acerca de la transformación digital en las Instituciones de Educación Superior, en adelante IES, en la pandemia por Covid-19.

Si bien, el proceso consultivo se realiza sobre el entorno educativo, es prudente mencionar que, al igual que en otros sectores, en la educación la economía se constituye como un elemento trasversal que pueden o no permitir el avance de la formación. Bajo este precepto la economia colombiana, presento un adecuado crecimiento en el año 2019, momento en el cual creció 3.3\% (DANE, 2020), indicador que está muy por encima al promedio de los países de América Latina y del Caribe, los cuales en promedio alcanzaron un incremento del $0.6 \%$ un punto por debajo de la proyección realizada para estos países al inicio del 2019, demostrando entonces un daño para la economia definiéndola como incapaz de superar los estándares que permiten darle continuidad a la lucha contra la pobreza.

Por su parte el estudio del DANE (2020) indica que al inicio del 2020 las proyecciones de crecimiento económico para Colombia eran optimistas estableciendo estándares cercanos al $3.7 \%$ anual y para países de América Latina y del Caribe en $2.1 \%$, crecimiento que, aunque se haya podido lograr debido a las condiciones del mercado, no lograron sopesar las necesidades sociales presentadas por la población, y mucho menos superar los impases que el desarrollo requiere.

Eventualmente, vale la pena mencionar algunos indicadores, que permiten identificar de mejor manera la situación de Colombia con respecto a la economía y las posibilidades de enfrentar la pandemia por Covid-19, tales como el consumo que vivió un desajuste de casi el $-5.1 \%$ o el PIB que tuvo un desajuste del -6.4 e inclusive la inversión que alcanzó niveles de $-22.1 \%$ (todos indicadores relacionados con el aporte al PIB). Ante ello, es prudente reconocer que el principal problema que se tuvo con respecto al entorno económico del país estuvo fuertemente relacionado con el confinamiento obligatorio, que se reconoce como una de las principales medidas adoptadas por la OMS y que de acuerdo con Fernández, Gómez y Perez (2020) permite evidenciar las disparidades sociales de las cuales son víctimas los colombianos que eventualmente se profundizan con el paso de la cuarentena nacional producto de la pandemia.

El aislamiento obligatorio que termina afectando de manera significativa las condiciones macro y micro económicas del país no solo por la imposibilidad de continuar con la cotidianidad que demarca comportamientos específicos y que remiten a la construcción cultural y social, impide la realización de acciones específicas de subsistencia, como es el trabajo. De acuerdo con el informe presentado por Alarcón (2020) en el país se alcanzaron a perder más de cuatro mil empleos, que significaron menor consumo, menor ahorro y disminución de la calidad de vida. Es preciso argumentar que ante esta problemática se presenta una disyuntiva bastante marcada, y es que, si bien una apertura del comercio podría implicar un incremento de la población trabajando, en la misma medida ello puede significar una mayor cantidad de contagios, ocupación de camas en las unidades de cuidados intensivos, y eventualmente una mayor cantidad de tiempo en restricción.

El sector laboral si bien se considera la piedra angular de la economía, precisamente porque se encuentra ampliamente ligado con las condiciones del mercado, con la entrada de la pandemia por Covid-19 termina afectando a otros sectores como el turismo, restaurantes y evidentemente la educación; de acuerdo con Carlos Estrada, director del Servicio Nacional de Aprendizaje, en adelante SENA, la deserción estudiantil, en respuesta a la pandemia ha 
incrementado en un $12 \%$ en la totalidad de los niveles de educación, siendo esta mayor a medida que pase el tiempo de confinamiento (Observatorio de la Universidad Colombiana , 2020).

$Y$ es que cuando se analiza la deserción como un problema que, aunque latente en la pandemia, es realmente estructural en Colombia, precisamente porque aun en la normalidad de acuerdo con el Ministerio de Educación Superior alcanza a ser del 8\% (Murillo-Vargas, Gonzalez, \& Urrego-Rodríguez, 2020), con la pandemia ha conseguido hacerse a otros determinante, como es la imposibilidad de conseguir conexión de internet estable para permanecer en las clases virtuales e inclusive la posibilidad de comprar un celular, computador o Tablet que permitiera la realización de las diferentes actividades.

De acuerdo con Valencia (2020) Colombia no se encuentra preparada para asumir los problemas relacionados con el sector educativo en pandemia precisamente porque para procurar una transición a una educación virtual con mayores niveles de mediación tecnológica, como es exigencia del Covid-19 existen problemas estructurales relacionados con la educación en sí misma y con el contexto tecnológico del país, asi pues en un primer momento, existe un escaso nivel de acceso real de la población a elementos tecnológicos básicos y conectividad, asi como también los docentes en la actualidad deberían adoptar los medios comunicativos como elementos trasversales en su profesión y quehacer educativo y finalmente los estudiantes deben poseer una mayor autonomía siendo conscientes de la relevancia de la formación para el progreso individual y colectivo.

Considerando el entorno digital que permea a Colombia como una barrera para alcanzar la digitalización de la educación, en miras a subsanar las necesidades expuestas por la pandemia por Covid-19, es necesario establecer que, aunque en el momento actual de acuerdo con los datos analizados por Katz, Jung y Collorda (2020) la penetración de internet en el país alcanza a ser de $71.40 \%$, lo cierto es que la mayor cantidad de población que se encuentra desprovista de internet es la población de escasos recursos y la población rural, que ha presentado un abandono significativamente marcado en los últimos años, demostrando nuevamente las disparidades sociales que permean el país, y que demarcan en buena medida el devenir del proceso formativo no se encuentran implicadas directamente con la presencia del virus.

Con el proceso de digitalización de la educación en Colombia, también se ha logrado identificar que, si bien la tenencia de dispositivos tecnológicos en el país alcanza a ser de más del $58 \%$ en las familias, refiriéndose a computadoras, como medios expresamente dedicados a la educación, lo cierto es que estos equipos no soportan la formación de varios miembros de la familia a la vez (Pinzón Ortiz, 2019), exponiendo la posibilidad no solo de tener disparidades a nivel nacional, regional sino también a nivel familiar, pues de una u otra forma, se prioriza la ocupación de estos equipos en miras de atender las necesidades de alguno de los miembros de la familia.

Otro problema subyacente a las condiciones digitales que logran considerarse como relevantes en el contexto nacional es necesario traer a colación el tipo de ocupación que se le está otorgando tanto al internet como a los dispositivos que se ocupan para dar uso a esta herramienta, en este sentido Katz, Jung y Collarda (2020) reconocen que en su mayoria los latinoamericanos realizan uso de computadoras y medios móviles como medios de comunicación y vinculación social, abandonando en buena medida las necesidades formativos, o rezagándolas, de hecho, de la totalidad de aplicaciones que son ocupadas 
por la población colombiana, solo el $50.73 \%$ se destinan a la educación.

Si bien los problemas en los hogares son bastante significativos en lo que respecta a la digitalización y virtualización de la educación y se encuentran relacionados con las posibilidades de acceso a internet y a implementos que permitan hacer la ocupación de este en pro de la formación de los colombianos, es el análisis de los actores que interfieren en el proceso educativo es necesario tener en cuenta también las condiciones mismas de las IES, en ese sentido, aunque con anticipación, la digitalización de la formación se había considerado una necesidad que tenían las instituciones debido a los procesos de acreditación, en muchos casos la implementación de las tecnologías de información y comunicación se reconoce como un proceso burocrático que no alcanza a llenar no solo las expectativas de la población sino también a subsanar las necesidades de movilidad (Cadelo Pérez, 2020).

Considerando al Banco Interamericano de Desarrollo (2020) se hace necesario reconocer que, aunque generalmente las universidades en Colombia no cuentan con procesos tecnológicos implementados, lo cierto es que los pocos esfuerzos realizados por algunas instituciones lograron abrir una brecha significativa en tiempos de pandemia, en ese sentido la universidad y la formación tiende a tener desigualdades entre los sujetos basada en las imposibilidades de las IES de implementar procesos de desarrollo enfocados en atender a la población educativa de manera virtual, eventualmente si bien la pandemia impulsa la creación de plataformas y redes comunicacionales, lo cierto es que el rezago se ve profundamente marcado de una universidad a otra y eventualmente de un producto a otro, teniendo en cuenta que el producto es el profesional como tal.

En la misma medida las IES se encuentra enfrentada a un problema sustancialmente relevante en cuanto al proceso de formación como tal, pues si bien los estándares evaluativos, y los desempeños que los estudiantes deben alcanzar con la formación presencial se encuentran adecuadamente sustentados en la educación presencial, lo cierto es que comprobar los saberes aprehendidos por el estudiante en la virtualidad resulta sustancialmente complejo sin un proceso que demuestre no solo conocimiento sino también metodologías diferenciadas $y$ experiencia por parte del docente (Grande de Prado, García Peñalvo, Corell, \& Abella-García, 2021).

A este punto vale la pena argumentar que para muchas IES es necesario, para garantizar la permanencia de los estudiantes la aprobación de las asignaturas, lo que no implica necesariamente que la calidad se encuentre asegurada, por el contrario la implementación de diferentes procesos de evaluación, destinados a mejorar las condiciones del estudiante en cuanto a la presentación de actividades o evaluaciones ha generado una flexibilización de la formación que afecta en buena medida la construcción de conocimiento significativo

Los docentes como actores transversales en el proceso de digitalización de la formación durante la pandemia, han tenido que generar procesos de formación relativamente fugaces apoyándose en buena medida en medios propios, capacitaciones vía video e inclusive algunas conferencias que han sido ofertadas de manera gratuita, de acuerdo con Castellanos y Otero (2020) el docente ha tenido la necesidad de triplicar su trabajo cambiando las maneras y didácticas que eran aplicadas en los espacios formativos, incluyendo en algunos casos la preocupación de medios físicos para que los estudiantes puedan o no asistir a clase, lo que implica necesariamente un desgaste que no corresponde para nada a su formación y mucho menos a su experiencia. 
De acuerdo con la UNESCO (2020) es esencial generar una capacitación constante del profesorado, precisamente considerando el acceso de la comunidad universitaria a recursos para la enseñanza aprendizaje, y procurar en la misma medida que la innovación se realice desde el escenario, no solo desde la pandemia sino del entorno propio de la educación la cual se encuentra revolucionándose constantemente bajo el paradigma de la modernidad.

El principal evento realizado entorno a los factores que inciden de manera directa en la digitalización de la educación es llevado a cabo por la UNESCO (2021) en la cual los participantes manifiestan la imperancia de los procesos de capacitación docente lo cierto es que se requiere de diferentes puntos, entre los cuales se encuentra la posibilidad de estructurar aseguramiento de la calidad en la enseñanza virtual, la equidad en el acceso y continuación de estudios superiores, precisamente porque las universidades generan dos de las tres partes del conocimiento científico, y ello supone la base para la innovación que tanto se requiere. Eventualmente no se trata solo de mejorar la conectividad sino también de fomentar la capacitación necesaria para ocupar la actualización e innovación a favor de los estudiantes.

La virtualidad, que es necesaria en tiempos de pandemia deja bastante claras algunas necesidades con las que conviven estudiantes y docentes en la cotidianidad, expresando una mayor cantidad de falencias, sin embargo, de manera generalizada se encuentra que, la formación docente en temas de digitalización en Colombia presenta diferentes problemas consistentemente fuertes precisamente porque aunque los saberes son bastante importantes en el tema de saberes estudiantiles, la dinámica pedagogica virtual y presencial son bastante diferentes, evidenciando que en algunos casos lo presencial se diferencia mucho de lo virtual (Rivera, 2020).

Para el caso, si bien los docentes se han considerado actores clave de los procesos de formación es preciso considerar que los estudiantes generan otros condicionantes desde sus condiciones particulares, que, si bien no obedecen de manera directa a la digitalización de la formación, si pueden estar influyendo en la educación. Precisando estos elementos alternos es prudente traer a colación el efecto psicológico que tiene el confinamiento en la capacidad de aprendizaje de los estudiantes, en este sentido muchos estudiantes conviven en ambientes que son poco favorables para adaptarse a los formatos virtuales, bajo las condiciones mismas de su hogar, la disposición de la red y el acceso a las tecnologías requeridas. De acuerdo con Valdivieso, Burbano y Burbano (2020) los estudiantes consideran el estado de ánimo durante la cuarentena un factor que afecto de forma negativa y fuerte la adquisición de nuevos conocimientos, en la misma medida existe una alta relación entre la adquisición de nuevos conocimientos y el afianzamiento de conceptos previos, la convivencia en su núcleo familiar antes de la cuarentena afectaba su estado de ánimo de forma negativa, situación que influye de manera significativa en el rendimiento académico.

No obstante, el confinamiento también proporciona diferentes espacios que son reflexivos para el estudiante y el docente, ello con la intención de incrementar la resiliencia y mejorar de manera consistente las estrategias de aprendizaje por medios virtuales que al momento actual no han tenido gran cabida en los entornos regionales y nacionales, para que en el futuro próximo puedan ser más competentes tanto en sus procesos de formación universitaria como en el campo laboral cuando su vida to requiera (Valdivieso, Burbano, \& Burbano, 2020) 
En el mismo sentido se reconoce que la existencia de una asociación moderada de la virtualidad, ha sido el punto de partida para la construcción de un estado de ánimo y la convivencia familiar del estudiante con el rendimiento académico, exponiendo a los estudiantes y familiares a un entorno que con anticipación no era propio de los contextos formativos. En la misma medida los estudiantes reconocen a la pandemia, el confinamiento y la implementación en el trabajo virtual realizado desde el hogar generan impactos negativos de tipo económico, emocional y social en sus demás familiares (Amaya-López, 2020).

Desde el analisis de los estudiantes y sus labores al interior de la academia se reconoce como una problemática la paralización de la investigación en el contexto de la pandemia, ello como consecuencia de los diferentes protocolos de distanciamiento social, que han visto comprometidos los medios, las condiciones y los sujetos de investigación, condicionando en buena medida la ocupación de las clínicas y laboratorio como medios para el logro de los objetivos, que en muchos casos genera incongruencia entre objetivos y metas investigativas. Eventualmente, las iniciativas que desde la investigación se han planteado fomentan de manera casi particular el desarrollo de iniciativas para superar barreras que afectan en buena medida el sistema funcional de la educación superior y sostenibilidad del sistema educativo, abandonando en muchos casos otras temáticas referentes a la construcción de conocimiento. (Chacín, González, \& Peñaloza, 2020)

Administrativamente si bien la generación de conocimiento resulta sustentar problemas en cuanto a la actividad misional de las IES lo cierto es que existe otro problema que presenta una mayor dificultad para las universidades y que se encuentra relacionado de manera directa con la sostenibilidad económica que estas deben tener, en este sentido se pueden enumerar diferentes factores, que desde la literatura se hacen aparentes.

Para iniciar tanto las universidades públicas como privadas en Colombia dependen en cuestión de ingresos de las matriculas que son pagadas por los estudiantes para el sostenimiento, sin embargo, debido a la alta deserción escolar, que se considera como una problemática sustancialmente relevante, lo cierto es que la cantidad de matrículas se han disminuido de manera significativa representando una disminución en la cantidad de dinero percibida por los establecimientos educativos; a esta situación se le suma que, en el caso específico de las universidades públicas, estas presentan problemas significativamente graves, pues dependen en buena medida de la cantidad de dinero que el Estado asigne para su funcionamiento, eventualmente debido a las condiciones propias del contexto de pandemia, los países deben hacer ajustes significativos en los presupuestos universitarios, creando una disyuntiva en cuanto a la situación economica y financiera, y para muchos casos se prioriza en buena medida la salud en lo que respecta al gasto en bienestar social (Observatorio de la Universidad Colombiana, 2021).

En Colombia las exigencias de la universidades públicas se encuentran orientadas hacia la promoción de la matricula cero, como una forma de frenar la deserción académica, lo que en ultimas termina implicando una mayor cantidad de recursos que deben ser asignados por parte del Estado el cual se encuentra con presión financiera dada la imposibilidad de conseguir fondos suficientes para soportar la actualización de los servicios de salud correspondientes con las mejoras requeridas para la atención de la pandemia, como el incremento de unidades $\mathrm{UCl}$ en los diferentes hospitales (Meritano, 2020).

Desde este mismo punto de vista el problema de la financiación afecta de manera consistente a las personas que se una u otra manera tienen 
beneficios estatales como son las becas, las cuales debido a la imposibilidad de continuar con su financiación deberían verse programadas para un tiempo futuro o suspendidas definitivamente. Para el caso de las universidades privadas en el entorno colombiano, la problemática esencial es que, aunque se cree que la mayoria de las personas que asisten a esta cuentan con los fondos suficientes para la financiación de sus estudios lo cierto es que en el momento actual son muchas las personas que dependen de sus trabajos o de préstamos para el pago de matrículas, bajo esta condición la disminución de los empleos y la no percepción de recursos, obliga a los estudiantes a retirarse de las universidades, dejando en estas un hueco fiscal bastante profundo (Chacón, 2021); de acuerdo con la ASCUN se encuentra una caída del $11.3 \%$ en matriculas de estudiantes sobre todo en el semestre 2 de 2020.

Una vez analizada la incidencia de los actores y los condicionantes económicos que han incidido en el proceso de formación de los estudiantes colombianos, es necesario enfocar el presente estudio en la transformación digital destinada al proceso educativo en pandemia. Bajo esta premisa es necesario considerar que de acuerdo con Ligarreto (2020) el acceso al internet se reconoce como fundamental para la implementación de un modelo educativo virtual que de resultado en las IES, en ese sentido el análisis de las cifras permite evidenciar que existen brechas difíciles de superar en cuanto a la financiación de las inversiones en conectividad, en este sentido el DANE indica que solo el $26 \%$ de la totalidad de los estudiantes de las zonas rurales cuentan con conectividad a internet, cifra diferentes a los estudiantes de las zonas urbanas quienes en un $86 \%$ cuentan con este servicio.

Adicionalmente a los planteamientos anteriores De Zubiria (2020) manifiesta en una columna realizada para Semana que tan solo el $4 \%$ de los municipios de Colombia cuentan con una buena conectividad, mientras que el $96 \%$ de las universidades deben lidiar con problemas de conectividad inclusive con los estudiantes que se encuentran en las zonas urbanas, ello defiendo la imposibilidad de generar un seguimiento personalizado, como es tradicional en las universidades presenciales, de las lecciones aprendidas y eventualmente de la evaluación y seguimiento que debe realizarse sobre los estudiantes a fin de verificar el cumplimiento de la calidad.

La visión de Valencia (2020) otorga una mirada todavía más preocupante sobre la situación vivenciada en el fomento de la educación desde el punto de vista virtual en Colombia, pues, si bien el incremento de la conectividad es necesario para potenciar la posibilidad de comunicación, lo cierto es que ello no supone necesariamente una alteración del modelo de enseñanza aprendizaje tradicional, precisamente porque para el entorno educativo la comunicación es básicamente mediada por la voz del docente y la escucha del estudiante, siendo las TIC una necesidad alterna o condicionada antes de la pandemia.

Las causas de la falta de integración de las TIC al entorno educativo superior, dependen de muchos factores, algunos de los cuales es la falta de concertación entre las administraciones académicas, con los docentes y estudiantes, las mismas actitudes de docentes y la siempre tensa relación existente entre los modelos pedagógicos tradicionales y los modelos emergentes (Cabero-Almenara \& LlorenteCejudo, 2020)

Finalmente es prudente reconocer que los documentos consultados permiten obtener una visualización generalizada sobre la problemática, sin embargo, los impactos específicos que puede tener la pandemia al interior del entorno educativo deben analizarse en periodos posteriores, precisando la posibilidad de realizar 
un comparativo antes y después de esta misma, con cifras consistentes sobre los determinantes de la formación. Desde el presente documento se realiza un acercamiento a estos condicionantes desde el punto de vista empírico y exploratorio.

\section{DISCUSIÓN}

La crisis sanitaria que trae la pandemia por Covid-19 implica no solamente la agudización de problemas sociales sino también la imposibilidad de continuar con dinámicas que desde el punto de vista educativo han logrado construir paulatinamente procesos de calidad. En este sentido la mayoria de los autores analizados coinciden en argumentar que en Colombia, aunque los procesos de digitalización son bastante similares a los de América Latina, las disparidades sociales siguen generando una problemática realmente relevante en el proceso de formación, que aunque permea en buena medida las condiciones de enseñanza aprendizaje en las clases presenciales noalcanza a tener una trascendencia como en el caso de la formación virtual, en donde la imposibilidad de conectarse e inclusive de hacerse a un medio de comunicación es una respuesta a la escases de recursos en las poblaciones rurales o menos favorecidas. Desde el presente apartado no solo se dará discusión a los hallazgos, sino que se presentaran algunas alternativas que el contexto internacional presenta para sobrellevar la crisis por la pandemia, como un proceso coyuntural que termina afectando las condiciones estructurales del país.

Así, es necesario considerar el estudio de Powell, van Dick y de Wall (2016) quienes establecen que en el momento actual, sin incluir la incidencia de la pandemia existen muchas empresas que se encuentran dedicadas a la generación de herramientas que pueden ser ampliamente ocupadas en la educación a fin de integrar las tecnologías de información y comunicación en el entorno formativo. Desde este punto de vista el principal aporte que realizan estas empresas es la generación de plataformas para la enseñanza y el aprendizaje, asi como diferentes estructuras comunicativas que no requieran de espacio físico en los computadores de los estudiantes y docentes, lo cual facilita la conectividad, pero también la interoperabilidad, las integraciones con terceros y el flujo de datos entre los sistemas. Se encuentra que, de manera generalizada en el mundo las universidades se estan adaptando a plataformas formativas y campus virtuales con la idea de generar un mayor andamiaje en la infraestructura digital.

No obstante, ante la necesidad de construir una infraestructura operativa digital desde la cual se manejen los diferentes procesos de las universidades es preciso considerar las implicaciones que ello tendría, en un primer momento vale la pena considerar que existe la posibilidad latente que se genere una privatización de la educación superior, lo que implicaría necesariamente el incremento de brechas sociales entre las personas que pueden hacerse al dinero para su formación y las que no, ello precisamente porque una transformación completa implica un costo supremamente alto tanto en la implementación de tecnologia como en la puesta en marcha de procesos de capacitación para que docentes y estudiantes en pro de fortalecer el uso adecuado de las diferentes herramientas provistas (Williamson \& Hogan, 2020). Proceso que difícilmente puede verse llevado a cabo por las instituciones de índole público, o por lo menos no si se considera el desbalance fiscal de la economía colombiana.

Aunque la privatización parezca la única salida para que la implementación de la digitalización se concrete de manera apropiada, en Colombia existen documentos oficiales que fomentan políticas públicas a favor de este proceso, para el caso específico el CONPES 3899 deja claro la necesidad de que la totalidad de colombianos tengan la posibilidad de tener acceso a conocimientos informativos y de las 
tecnologías de información, que se encuentran a la vanguardia de los procesos de desarrollo en el mundo, así mismo se presenta la posibilidad de contar con un computador por cada casa y acceso a internet constante. El Gobierno Nacional también ha manifestado esta necesidad desde la Ley 1955 de 209 en la cual se reconoce la necesidad de diseñar e implementar planes, programas y proyectos que promuevan de manera prioritaria el acceso y servicio universal a las TIC

Bajo los mismos condicionantes es necesario reconocer que, la transformación que se realice en cuanto a la digitalización de la educación debe tener como un condicionante el cumplimiento de los objetivos en los ámbitos: pedagogía y desarrollo curricular, asi como los diferentes debates que se tendrían que realizar, considerando el papel crítico de la educación superior en los desafíos que tienen las regiones y el país en general.

Desde la pandemia y los problemas que esta ha traído para el sector educativo es prudente mencionar la necesidad de generar procesos de formación para docentes y directivos en el uso y manejo de las plataformas digitales, precisando en este punto que el triduo (Estado, Instituciones y Docentes-Estudiantes) debe estar completo y sobre todo trabajar mancomunadamente para continuar proyectando la calidad académica como un elemento trasversal en el desarrollo del país. En este sentido es prudente que la transformación digital se vea aparejada de una transformación profunda de actividades, organizaciones, procesos, competencias y modelos, aprovechando la tipificación completa de problemas que han sido aparentes con la pandemia por Covid-19 (Castro Benavides, Tamayo Arias, Arango Serna, Branch Bedoya, \& Burgos, 2020).

La transformación digital al interior de las Instituciones de Educación Superior debe tener en cuenta que los retos que se le presentan no solo contienen una modificación en la infraestructura comunicativa lo que incluye procesos de conectividad, sino también la transformación y el paso de una pedagogía netamente tradicional a una pedagogía que comprende los cambios generacionales $y$ se alinea en buena medida con las nuevas tendencias del mundo moderno.

Los diferentes documentos que fueron analizados coinciden en argumentar que, aunque la contingencia representa un condicionante bastante problemático en el entorno nacional, también se permitió crear oportunidades y ventanas para poner en la agenda pública la necesidad de una transformación orientada a lo digital de manera urgente, para lo cual es obligatorio y urgente generar procesos de formación en competencias digitales tanto en los estudiantes como en los profesores, asi como también la necesidad de dotar de equipos de cómputo y acceso a internet a las personas que se encuentran en zonas rurales y en poblaciones que han sido rezagadas históricamente, en concordancia también se debe garantizar el acceso a todos los servicios públicos a fin de generar condiciones contextuales apropiadas, e ir paulatinamente incluyendo en la formación tradicional la ocupación de redes de información, la necesidad de la formación a distancia y el desarrollo de servicios básicos de información (Safiullin \& Akhmetshin, 2019).

La modificación de los condicionantes propios del entorno educativo con miras a generar un proceso de formación digitalizado requiere en el mismo sentido modificaciones profundas en la cultura, la gestión del conocimiento y la apertura del conocimiento. En este sentido es prudente reconocer que la transformación no solo requiere realizarse precisando la necesidad de mejorar de manera consistente la forma en la que se asume la pandemia, sino como se asumen en general los cambios frente a las modificaciones que provee el entorno, en ese sentido es 
necesario que se provean pensadores agiles, con recursos de calidad y plataformas abiertas para compartir el conocimiento y la formación de conocimientos de manera más activa. De esta manera la capacitación, la formación y la gestión del capital humano se reconocen como aspectos clave en la era digital (Sirotkina, Meshcheryakova, Syshchikova, Filatova, \& Greshonkov, 2019).

Considerando las necesidades latentes de formación es preciso reconocer que al interior de los documentos analizados aparece de manera constituyente el termino de innovación educativa el cual desde su proceso de adaptación al entorno ha presentado una alternativa viable a la generación de nuevos conocimientos, procesos, productos y servicio que estén orientados a mejorar las practicas institucionales en la Educación Superior. No obstante, como concepto la innovación educativa tiene en cuenta diferentes condicionantes, el primero de los cuales es la formación docente buscando que este innove en procesos formativos con la intención de incluir cada vez más contenido virtual, y consecuentemente estar a la par de las condiciones contextuales que son presentadas por los estudiantes (Ramírez-Montoya, 2020).

La innovación tecnológica se reconoce como un factor transversal en la generación de fomento educativo digital precisamente porque esta permite la ubicación de recursos y plataformas que estén prestos a fomentar el aprendizaje, específicamente en áreas que requieran de laboratorios y simuladores. Bajo el mismo sentido, es prudente concretar propuestas para generar innovación en la investigación, que como se ha visto se suspendió definitiva o momentáneamente debido a las diferentes cuarentenas que imposibilitaron el traslado de los investigadores a diferentes puntos donde se encontraban o los sujetos de investigación o los implementos necesarios para la construcción de conocimiento, eventualmente Colombia reconoce la necesidad de incorporar este tipo de procesos en la búsqueda de probar aciertos y desaciertos con la intención de continuar con la mejora no solo de la educación sino de las condiciones totales del país (Montoya \& Ocando, 2020).

Es importante demarcar que el proceso de transformación educativa debe verse aparejado de la intención de cada uno de los círculos sociales que se ven impactados de una $u$ otra manera con la educación aporten de manera significativa a la construcción de una transformación orientada al mejoramiento de las condiciones educativas de los jóvenes en las universidades, ello se podría definir como un pacto solidario en donde desde una mirada flexible realizada sobre el contexto colombiano es absolutamente necesario generar procesos de formación amparados en realidades sociales que coadyuven a los procesos formativos a ser mucho más autónomos. Esta idea se ve soportada en buena medida por los planteamientos expuestos por Branch, Burgos, Serna y Ortega (2020) quienes sugieren que es necesario trabajar la innovación desde la cultura misma, presenciando la creación de nuevos espacios de evaluación, reflexión, rediseño de procesos y diseño de propuestas como la acción clave para el desarrollo de nuevos enfoques educativos.

\section{CONCLUSIONES}

La pandemia por Covid-19 demostró la falta de preparación que tiene el país para asumir no solo la educación virtual como como un elemento trasversal en la formación de los colombianos, sino que también logro precisar las disparidades sociales marcadas entre la población de escasos recursos y los estratos medio altos, asi como también las diferencias entre la población rural y urbana en lo referente a la conexión a internet y los medios para la generación de investigación y construcción 
de conocimiento desde la autonomía o la guía asistida por medios virtuales.

Colombia contaba al momento de la pandemia para una muy baja capacidad de adaptación a las dinámicas impuestas por el mundo moderno y por las tecnologías de información y comunicación. Lo que desencadeno necesariamente en un impacto bastante fuerte y la improvisación en medios de comunicación que permitieran continuar con los modelos tradicionales de formación basados en las clases magistrales.

Desde el punto de vista económico el impacto que en mayor medida afecto a las universidades e IES fue la pérdida de empleos, que alcanzo a provocar un incremento de la tasa del desempleo hasta el $15 \%$, incrementando en el proceso la pobreza absoluta llegando al nivel de $40 \%$, y demostrando en el camino un retroceso en el país de casi u siglo.

La educación en Colombia que se ve apabullada por la crisis pandémica profundizo sus problemáticas en lo que respecta a cobertura y calidad. Considerando que la cobertura en el momento ya no se analiza desde la posibilidad de que los docentes e instituciones lleguen a una mayor cantidad de poblaciones, sino desde la posibilidad e imposibilidad de contar con una adecuada conexión a internet y un equipo de cómputo que le permita al estudiante estar a la par no solo de sus compañeros sino también de los docentes. En lo que respecta a la calidad, como condición intrínseca del proceso formativo es prudente mencionar que con el paso de la pandemia y de la imposibilidad de planear procesos de formación digitalizado se ha conseguido retrasar procesos no solo de formación sino también de investigación y construcción de conocimiento, factores que se vieron retrasados.

La educación digital ha ocupado un papel protagónico no solo en el entorno de la pandemia, sino en el contexto modernizado, debido a lo cual es preciso que se puedan generar estrategias de cobertura, mejorando los contenidos pedagógicos y sobre todo la ocupación de estrategias pedagógicas que permitan la resolución de casos, aprender nuevas herramientas y pasar de una educación tradicional a una educación transformada.

Es prudente mencionar la necesidad de cambiar el paradigma que se tiene acerca de implementar las TIC a la formación tradicional, considerando la puesta en marcha de modelo en donde la digitalización se considere un elemento fundamental para la promoción de conocimiento, y no solo un complemento en tiempos de distanciamiento. Específicamente en las IES concibiendo la ocupación constante de nuevas herramientas de manera paulatina sin las implicaciones de la obligatoriedad, promueve la adaptación y entendimiento de las herramientas, que se considera el fin último de ocupación de las mismas.

Es necesario que se considere la incidencia que tienen las empresas dedicadas a la digitalización en la promoción de procesos destinados al mejoramiento de las condiciones de formación de las universidades, precisamente porque son estas un factor de la cadena de aprovisionamiento que puede incrementar el impacto en la digitalización de las aulas, vale la pena pensar a estas como aliados para la construcción de un nuevo contexto y de una nueva formación.

La pandemia permitió en el mismo sentido identificar de manera consistente una ventana de oportunidad para poner en marcha procesos de digitalización que llevaban un rezago importante en Colombia, situación por la cual en el campo de la educación esta debe vislumbrarse como una oportunidad para el cambio y el crecimiento.

El trabajo mancomunado de los actores que estan presentes en la generación de conocimiento 
y la creación del mismo se establece como un impulso fundamental en la transformación digital de la educación superior, de ahí que, se deberían tener bien claros los roles y asignar efectivamente las tareas que cada uno de estos actores debe realizar.

\section{REFERENCIAS BIBLIOGRÁFICAS}

Alarcón Suárez, R. D. (2020). La educación digital en Colombia en tiempos de Covid 19 y su impacto en las organizaciones educativas. Universidad Militar de Colombia.

Amaya-López, L. F. (2020). El papel del vínculo psicoafectivo familiar en el tránsito de la educación presencial a la educación virtual y remota en el marco de la emergencia sanitaria por la Covid-19. . Búsqueda, 7(24).

Argudo, M. d. (2019). Educacion con tecnologia en una pandemia.Breve analisis. Journal of Chemical Information and Modeling .

Banco Interamericano de Desarrollo. (2020). La Educación Superior en Tiempos de Covid. BID.

Branch, J. W., Burgos, D., Serna, M. D., \& Ortega, G. P. (2020). Digital Transformation in Higher Education Institutions: Between Myth and Reality. In Radical Solutions and eLearning (pp. 41-50). Springer, Singapore.

Cabero-Almenara, J., \& Llorente-Cejudo, C. (2020). Covid-19: transformación radical de la digitalización en las instituciones universitarias. . Campus Virtuales, 9(2), 25-34.

Cadelo Pérez, A. (2020). La adaptación de la escuela al COVID-19, un paso hacia la digitalización.
Castellanos, M., \& Otero, J. (2020). Diálogos para la transformación digital. Educación desde lo presencial hasta lo virtual. Brecha Cero.

Castro Benavides, L. M., Tamayo Arias, J. A., Arango Serna, M. D., Branch Bedoya, J. W., \& Burgos, D. (. (2020). Digital transformation in higher education institutions: A systematic literature review. . Sensors, 20(11), 3291.

Chacín, A. J., González, A. I., \& Peñaloza, D. W. (2020). Educación superior e investigación en Latinoamérica: Transición al uso de tecnologías digitales por Covid-19. . Revista de ciencias sociales, 26(3), 98-117.

Chacón, M. (2021). Desplome en número de matriculados en universidades por la pandemia. El Tiempo.

DANE. (2020). Indicadores económicos. Presidencia de la República.

De Zubiria, J. (2020). La educación en tiempos de cuarentena. Semana.

Fernández-Sánchez, H., Gómez-Calles, T. J., \& Pérez, M. P. (2020). Intersección de pobreza y desigualdad frente al distanciamiento social durante la pandemia COVID-19. . Revista Cubana De Enfermería, 36.

Grande de Prado, M., García Peñalvo, F. J., Corell, A., \& Abella-García, V. (. (2021). Evaluación en Educación Superior durante la pandemia de la COVID-19. . Campus Virtuales, 1(10), 49-58.

Katz, R., Jung, J., \& Callorda, F. (2020). El estado de la digitalización de América Latina frente a la pandemia del COVID-19. Scioteca. 
Ligarretto, E. (2020). Educación virtual: Realidad o ficción en tiempos de pandemia. . Pesquisa Javeriana, 90, 20-32.

Mckinsey, R. (2020). The use of digital technologies by small and medium enterprise during COVID-19. International Journal of information.

Meritano, A. (2020). Colombia-La crisis también golpeo a la Universidad Nacional. Diarío Juridico.

Montoya, M. S., \& Ocando, J. L. (2020). Revisión sistemática de métodos mixtos en el marco de la innovación educativa. - Comunicar: Revista científica iberoamericana de comunicación y educación, (65), 9-20.

Murillo-Vargas, G., Gonzalez, C. H., \& UrregoRodríguez, D. (2020). Transformación del Sistema de Aseguramiento de la Calidad de la Educación Superior en Colombia 2019-2020. . Educación y Humanismo, 22(38).

Observatorio de la Universidad Colombiana . (2020). Pandemia ha causado una deserción del $60 \%$ de aprendices del SENA. Universal .

Observatorio de la Universidad Colombiana. (2021). Covid agravó situación financiera de Uniandes, que ya venía mal desde antes. Universidad.

Pinzón Ortiz, J. E. (2019). Modelo de Interacción inteligente del Reglamento del Congreso de Colombia para dispositivos móviles "MIRC".

PNUD. (2019). Cómo la COVID-19 ha acelerado la transformación digital. Santiago de Chile: Publicaciones PNUD.
Poell, T., van Dijck, J., \& De Waal, M. (2016). De platformsamenleving: Strijd om publieke waarden in een online wereld (p. 180). . Amsterdam university press.

Ramírez-Montoya, M. S. (2020). Transformación digital e innovación educativa en Latinoamérica en el marco del COVID-19. . Campus Virtuales, 9(2), 123-139.

Rivera, J. J. (2020). El Efecto del COVID-19 en la Economía y la Educación: Estrategias para la Educación Virtual de Colombia. . Revista Scientific, 5(17), 280-291.

Safiullin, M. R., \& Akhmetshin, E. M. (2019). Digital transformation of a university as a factor of ensuring its competitiveness. . International Journal of Engineering and Advanced Technology, 9(1), 73877390.

Sirotkina, N., Meshcheryakova, M., Syshchikova, E., Filatova, M., \& Greshonkov, A. (2019). Directions of the transformation of companies and universities in the digital economy. In Proceedings of the 33rd International Business Information Management Association Conference. IBIMA 2019.

UNESCO. (2020). IMPACTO DE LA CRISIS DEL CORONAVIRUS COVID-19 EN LAS IES: RESPUESTASALAEMERGENCIA Y TRANSFORMACIÓN DIGITAL DE LA EDUCACIÓN SUPERIOR. IESALC.

Valdivieso, M., Burbano, V., \& Burbano, A. (2020). Percepción de estudiantes universitarios colombianos sobre el efecto del confinamiento por el coronavirus, $y$ su rendimiento académico. Revista espacios. 
Valencia, J. (2020). Covid-19, TIC y educación ¿Por qué no estamos preparados? Universidad del Norte.

Williamson, B., \& Hogan, A. (2020).

Commercialisation and privatisation in/ of education in the context of Covid-19. 